\title{
Article
}

\section{Burden of Diabetic Retinopathy amongst People with Diabetes Attending Primary Care in Kerala: Nayanamritham Project}

\author{
Sobha Sivaprasad ${ }^{1, *(1)}$, Vasudeva Iyer Sahasranamam ${ }^{2}$, Simon George ${ }^{2}\left(\mathbb{D}\right.$, Rajeev Sadanandan $^{3}(\mathbb{D}$, \\ Bipin Gopal ${ }^{4}$, Lakshmi Premnazir ${ }^{4}$, Dolores Conroy ${ }^{5}$, Jyotsna Srinath ${ }^{6}$, Radha Ramakrishnan ${ }^{5}$, \\ Sundaramuthil Murukaiah Vijayanand ${ }^{4}$, Raphael Wittenberg ${ }^{7}$ and Gopalakrishnan Netuveli ${ }^{6,+}$ \\ on behalf of the Nayanamritham Project Collaborators
}

Citation: Sivaprasad, S.;

Sahasranamam, V.I.; George, S.;

Sadanandan, R.; Gopal, B.; Premnazir,

L.; Conroy, D.; Srinath, J.;

Ramakrishnan, R.; Vijayanand, S.M.;

et al. Burden of Diabetic Retinopathy amongst People with Diabetes Attending Primary Care in Kerala: Nayanamritham Project. J. Clin. Med. 2021, 10, 5903. https://doi.org/ $10.3390 / \mathrm{jcm} 10245903$

Academic Editor: Kyung Chul Yoon

Received: 2 November 2021

Accepted: 10 December 2021

Published: 16 December 2021

Publisher's Note: MDPI stays neutral with regard to jurisdictional claims in published maps and institutional affiliations.

Copyright: (c) 2021 by the authors. Licensee MDPI, Basel, Switzerland. This article is an open access article distributed under the terms and conditions of the Creative Commons Attribution (CC BY) license (https:// creativecommons.org/licenses/by/ $4.0 /)$.
1 NIHR Biomedical Research Centre, Moorfields Eye Hospital NHS Foundation Trust, London EC1V 2PD, UK

2 Regional Institute of Ophthalmology, Thiruvananthapuram 695035, India; drsahasranamam@gmail.com (V.I.S.); simongeorge1986@yahoo.com (S.G.)

3 Health Systems Transformation Platform, New Delhi 110070, India; rsadanandan@gmail.com

4 Directorate of Health Services, Thiruvananthapuram 695035, India; bipingopal@yahoo.com (B.G.); lakshmipremnazir08@gmail.com (L.P.); smvijayanand@yahoo.com (S.M.V.)

5 UCL Institute of Ophthalmology, London EC1V 9EL, UK; d.conroy@ucl.ac.uk (D.C.); r.ramakrishnan@ucl.ac.uk (R.R.)

6 Institute for Connected Communities, University of East London, London E16 2RD, UK; j.srinath@uel.ac.uk (J.S.); g.netuveli@uel.ac.uk (G.N.)

7 Centre for Health Service Economics and Organisation, Nuffield Department of Primary Care Health Sciences, University of Oxford, Oxford OX2 6GG, UK; raphael.wittenberg@phc.ox.ac.uk

* Correspondence: sobha.sivaprasad@nhs.net

+ Membership of the Nayanamritham Project Collaborators is provided in the Acknowledgments.

Abstract: Background: The burden of diabetic retinopathy (DR) in people attending the public health sector in India is unclear. Thirty percent of the population in India is reliant on public healthcare. This study aimed to estimate the prevalence of DR and its risk factors in people with diabetes in the non-communicable disease registers who were attending the family health centres (FHCs) in the Thiruvananthapuram district in Kerala. Methods: This cross-sectional study was conducted over 12 months in 2019 within the framework of a pilot district-wide teleophthalmology DR screening programme. The age- and gender-adjusted prevalence of any DR and sight-threatening DR (STDR) in the whole sample, considering socio-demography, lifestyle and known clinical risk groups, are reported. Results: A total of 4527 out of 5307 (85.3\%) screened in the FHCs had gradable retinal images in at least one eye. The age and gender standardised prevalence for any DR was $17.4 \%$ (95\% CI 15.1, 19.7), and STDR was 3.3\% (95\% CI 2.1,4.5). Ages 41-70 years, males, longer diabetes duration, hyperglycaemia and hypertension, insulin users and lower socio-economic status were associated with both DR outcomes. Conclusions: The burden of DR and its risk factors in this study highlights the need to implement DR screening programs within primary care to reduce health inequality.

Keywords: diabetic retinopathy; Kerala; India; diabetes; screening; socio-economic status; risk factors

\section{Introduction}

Kerala has one of highest prevalence of diabetes amongst all states in India. Approximately $10 \%$ of adults in Kerala are estimated to have type 2 diabetes (T2DM) [1,2]. The state has undergone significant economic and health transitions over the last 30 years [3]. However, the triad of increasing wealth, improved lifestyle and reduced physical activity has contributed to the rising prevalence of T2DM and its complications [2].

Diabetic retinopathy (DR) is a common complication of diabetes and an avoidable cause of blindness [4]. Currently, approximately $4.5 \%$ of blindness in India is due to sightthreatening DR (STDR) [4,5]. Early identification and treatment of STDR reduces the risk of 
blindness. As a largely asymptomatic condition, people with diabetes have to be screened regularly for DR [6]. Systematic screening for DR in India is in its infancy.

Most affluent people can access private healthcare in India and are therefore more likely to be screened for DR. In comparison, people who are socially disadvantaged, economically challenged and systemically marginalised rely mainly on the public health system [7]. In the absence of systematic DR screening in the public health system, the prevalence of DR in people who attend the public health system is not known. As primary care infrastructure is underdeveloped in India, data from primary care are scanty.

The government of Kerala has significantly revamped the primary care in the public health system by introducing family health centres (FHCs) with electronic health records (eHealth) $[8,9]$. For people with diabetes, a comprehensive diabetes care plan was initiated about five years ago, and it includes screening for all complications of diabetes except DR [10]. Therefore, implementing a DR screening programme within the primary care not only provides a complete preventive medicine plan for people with diabetes but also contributes to achieving some of the Sustainable Development Goals (SDG) [11]. The SDG 3 is to achieve health and well-being for all people, and SDG 7 is to reduce inequalities by 2030 .

The burden of DR and its risk factors in Kerala will also provide information on resource allocation for systematic DR screening in the primary care in the public health system.

In this cross-sectional study, we report the prevalence of any DR, STDR and referable retinopathy and associated risk factors in newly screened people with diabetes within the FHCs in the Thiruvananthapuram district in Kerala.

\section{Methods}

\subsection{Study Design and Setting}

The Government of Kerala collaborated with Moorfields Eye Hospital in the ORNATE India project funded by the Global Challenge Research Fund and United Kingdom Research and Innovation to set up the Nayanamritham project [12]. This pilot teleophthalmology DR care pathway was implemented for people utilising the public health system. Screening for DR was offered for people with diabetes attending the FHCs in the Thiruvananthapuram district and treatment for STDR delivered in secondary care hospitals [13]. This is a cross-sectional study of all individuals with diabetes registered in the non-communicable diseases (NCD) register who participated in the teleophthalmology DR screening program of Nayanamritham project in the 16 FHCS of Trivandrum District, Kerala over 12 months in 2019. Each FHC independently maintained a NCD register.

\subsection{Participants}

Individuals with diabetes aged 30 years or above were identified from the NCD register and invited to participate in the DR screening program when they attended the FHCs for their diabetes care.

\subsection{Data Acquisition}

A study-specific questionnaire was administered to each patient by data entry operators at each FHC. Individual data included demographics, education, lifestyle (smoking, alcohol and physical activity), family history, blood pressure, body mass index (BMI) and waist circumference (WC). The participants also answered questions on their perception of their quality of life and vision. Self-reported history of macrovascular and microvascular complications, coronary heart disease, stroke, diabetic neuropathy and diabetic kidney disease was also collected. On the day of screening, blood pressure and either random blood glucose (RBG) or fasting blood glucose (FBG) were measured, and urine samples were tested for presence of albumin. 


\subsection{Diabetic Retinopathy Screening Protocol}

When people with diabetes attended the FHCs for their regular diabetes care, both eyes were dilated with $1 \%$ tropicamide before retinal photography. Retinal images were captured by existing non-ophthalmic-trained primary care staff using indigenous smartphoneenabled retinal cameras (Remidio Fundus on Phone (FOP; Remidio Innovative Solutions Pvt. Ltd., Bengaluru, India) fixed on a frame and used as a tabletop device. This camera is compliant with European Conformity (CE marked) and the Health Insurance Portability and Accountability Act (HIPAA) and has been previously validated and used in several countries [14,15]. The FHC staff (nurses and doctors) were trained on the study protocol, the DR screening and referral pathway, mydriasis, capturing good quality retinal images and DR grades. Certificates of completion of training were issued by the University of East London, United Kingdom.

The retinal images were transferred through a newly established picture archiving and communication system (PACS) to the Regional Institute of Ophthalmology (RIO), the tertiary ophthalmic centre located in Thiruvananthapuram. For each eye, two images were taken and for most individuals images were taken for both eyes. The retinal images were graded at RIO by two certified graders, supervised by retinal specialists. The retinal graders were certified as accredited graders following completion of an online DR screening course, offered by the Gloucestershire NHS Foundation Trust [16].

The retinal photographs were first graded for quality of images based on the proportion of retina visible in the image available for grading. Four categories were used to describe gradeability: $100 \%$ gradable, $75 \%$ gradable, $50 \%$ gradable and less than $50 \%$ gradable, the latter of which was defined as ungradable.

For gradable images, the severity of DR was graded according to the International DR severity grading as no DR, mild non-proliferative diabetic retinopathy (NPDR), moderate NPDR, severe NPDR and proliferative diabetic retinopathy (PDR) [17]. PDR was further classified into stable treated and active PDR requiring laser photocoagulation and advanced diabetic eye disease (vitreous haemorrhage, tractional retinal detachment, rhegmatogenous retinal detachment, iris or angle neovascularisation, neovascular glaucoma and blindness due to DR. Diabetic macular oedema (DMO) was graded as per the definitions of clinically significant DMO as absent or present [17]. People with ungradable retinal images were also referred to secondary care for further evaluation.

\subsection{Outcomes}

The prevalence of the following in the study sample adjusted for age and gender was analysed.

1. Any DR was defined as presence of any grade of NPDR, PDR or DMO in at least one eye of an individual.

2. Referable retinopathy included severe NPDR, PDR or DMO in any or both eyes of an individual.

3. DMO in any or both eyes of an individual.

4. STDR was defined as presence of PDR and/or DMO.

Secondary analysis included prevalence of these outcomes in each FHC as well as in sub-populations defined by socio-demographic, lifestyle and clinical factors.

\subsection{Sample Size}

Our sample consisted of all those who attended DR screening at the 16 FHCs during the study period. Using an estimated DR prevalence figure of $10 \%$, we estimated, using Cochran's sample size formula, that we would need a sample of 3458 individuals to estimate that level of prevalence with $1 \%$ error. To offset any loss to the sample due to ungradable images and incompleteness of data collected by newly trained data operators, we planned to recruit at least 5000 people with diabetes. 


\subsection{Statistical Analysis}

Since the sample was drawn from NCD registers maintained independently in each FHC, there was the potential for clustering effects in the sample. We accounted for any clustering effect in the analyses by setting up the data as complex survey data with each FHC as the primary sampling unit.

\subsection{Estimation of Prevalence of $D R$}

The prevalence was calculated adjusted by age and gender. As the standard errors from direct standardization would not account for clustering, we used predicted marginal probabilities for the purpose of prevalence calculation (Stata command margins after logistic regression with age and gender and command contrast for testing statistical significance between categories). We report the prevalence for the whole sample screened for subpopulations defined by known risk factors and for each of the FHCs. We also fitted a multivariable logistic regression to test the strength of association of the risk factors to the outcomes.

To identify if the FHCs cluster was based on the prevalence of any DR and STDR we did a k-means cluster analysis of the prevalence figures from 15 FHCs. This sample size might be adequate based on Formann's rule of thumb, $2^{p}$, where $p$ is the number of variables (two in our case) [18].

\subsection{Risk Factor Analysis}

\subsubsection{Socio-Demographic Variables}

Age was categorised into the following groups: 31-40 years, 41 to 50 years, 51 to 60 years, 61 to 70 years or more than 70 years. Other variables considered were gender, education (none, primary, secondary or graduate and higher) and occupation (not working, housewife, retired, unskilled worker, skilled worker, professional and self-employed). We created a binary variable for self-reported income above and below INR 600, the sample median income.

\subsubsection{Diabetes Variables}

These included parental history of diabetes (none, either one of the parents or both parents having diabetes), duration of diabetes (less than 4 years, 4 to 9 years or more than 9 years since first diagnosis of diabetes), whether insulin was used in the treatment or not, having at least one complication of diabetes pre DR screening and a categorical variable indicating uncontrolled diabetes based on fasting blood glucose (FBG $\geq 7 \mathrm{mmol} / \mathrm{L}$ ) or random blood glucose (RBG $\geq 11.1 \mathrm{mmol} / \mathrm{L})$.

\subsubsection{Behavioural Risk Factors and Covariates}

Behaviours relating to smoking, physical activity and diet were combined to create a healthy lifestyle score. Smoking was scored as 0 for smokers, 0.5 for ex-smokers and 1 for non-smokers. Physical activity was scored as 1 for those participating in activities of moderate or severe intensity and 0 otherwise. Dietary habits were scored based on a checklist of five unhealthy dietary habits, namely intake of salty snacks, fried snacks, fruits less than once a day, vegetables less than once a day and meat and poultry more than twice a day. The diet score was 0 if three or more items were checked, 0.5 if two items were checked and 1 if one or no item was checked. Healthy lifestyle score was a summative score based on these three scores and further dichotomised at the median.

\subsubsection{Clinical Risk Factors and Covariates}

Obesity status was categorised according to the Asian criteria, with a body mass index (BMI) 18.5-22.9 as normal, overweight as $23-24.9$ and obese as $\geq 25$. Waist circumference was dichotomised according to the above WHO-recommended cut off points (for women: $\geq 88 \mathrm{~cm}$ and for men: $\geq 102 \mathrm{~cm}$ ) [19]. Hypertension status of each subject was classified by systolic blood pressure (SBP) according to the 2017 Guidelines for High Blood Pressure 
in Adults from the American College of Cardiology and American Heart Association (Hypertension stage $1 \mathrm{SBP} 130-139 \mathrm{mmHg}$, Hypertension stage $2 \mathrm{SBP}>139 \mathrm{mmHg}$ ) [20]. We also noted the presence of other complications of diabetes such as neuropathy and chronic kidney disease. If the patient reported that they were told they had DR previously, it was also noted. Analyses were performed using STATA 15.1 (StataCorp LLC, College Station, TX, USA).

\section{Results}

The study complied with the Declaration of Helsinki and was approved by The Indian Council of Medical Research (ICMR)/Health Ministry Screening Committee (HMSC / (20180551) dated 13/03/2019. Written informed consent was obtained from each participant.

A total of 5307 individuals with diabetes in the NCD registers at the 16 FHCs were screened. All those who did not meet the eligibility criteria or had missing primary outcome data were excluded. One FHC (Kadakkampally) was excluded due to outlying results which could not be validated by re-examination (Table 1). The final sample size was 4527 $(85.3 \%)$. The flow diagram (Figure 1) shows the derivation of the study sample size.

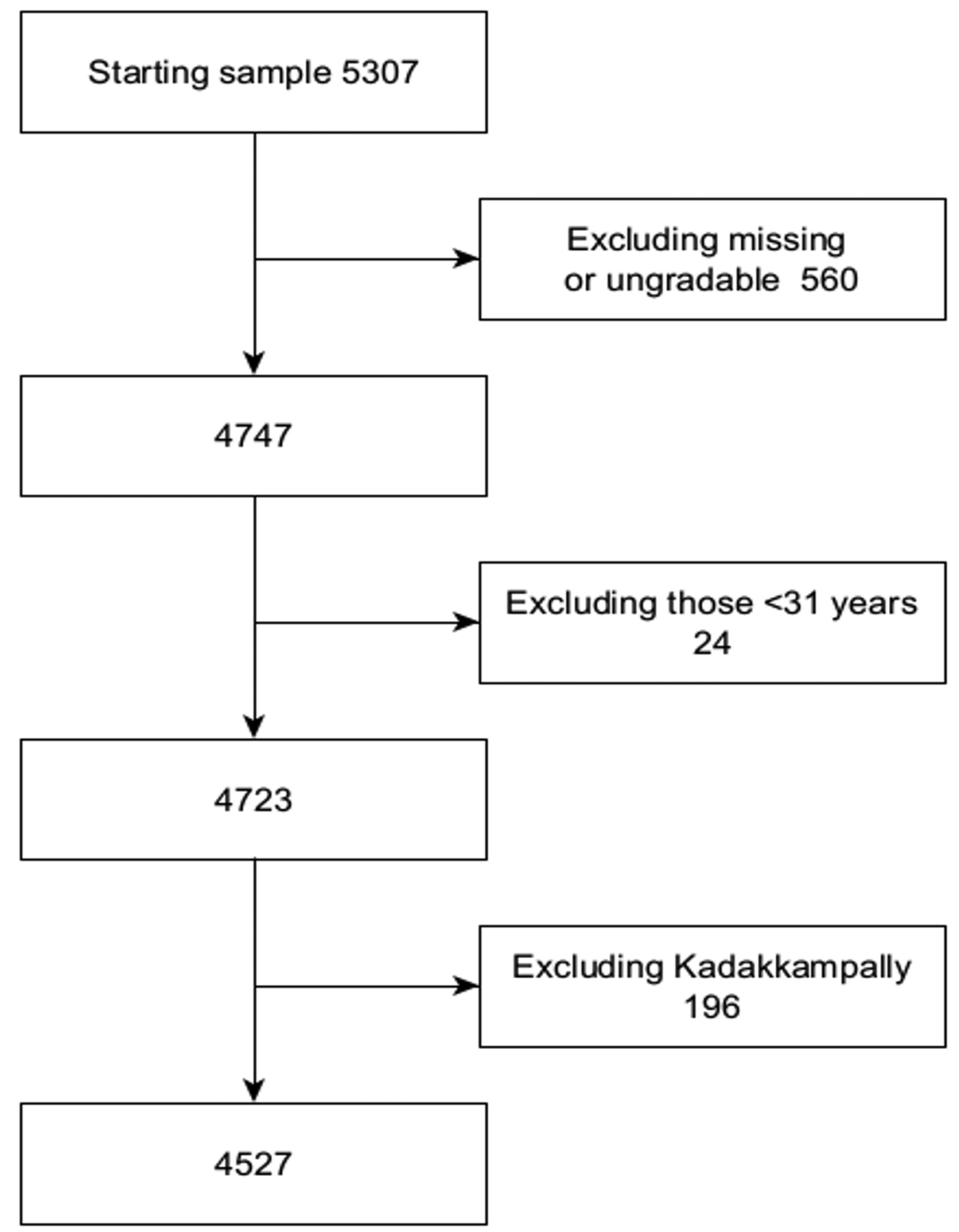

Figure 1. Flow diagram showing derivation of the study sample size. 
Table 1. The representativeness of the sample at each FHC.

\begin{tabular}{|c|c|c|c|}
\hline Family Health Centre (FHC) & Population Served by FHC & $\begin{array}{c}\text { Numbers }(\%) \text { or People with } \\
\text { Diabetes }\end{array}$ & $\begin{array}{c}\text { Screened Population } \\
\text { N (\%) } \\
5307\end{array}$ \\
\hline Amachal & 42,240 & $2768(6.5 \%)$ & $140(5 \%)$ \\
\hline Aruvikkara & 37,555 & $4017(10.6 \%)$ & $412(10.2 \%)$ \\
\hline Balaramapuram & 37,185 & $4202(11.3 \%)$ & $538(12.8 \%)$ \\
\hline Chemmaruthi & 37,188 & $4122(11.1 \%)$ & $323(7.8 \%)$ \\
\hline Kadakampalli * & 37,233 & $596(1.6 \%)$ & $306(51.3 \%)$ \\
\hline Karakulam & 68,408 & $1864(2.7 \%)$ & $353(18.9 \%)$ \\
\hline Keezhattingal & 29,522 & $842(2.8 \%)$ & $264(31.3 \%)$ \\
\hline Kilimanoor & 24,894 & $1632(6.5 \%)$ & $390(23.8 \%)$ \\
\hline Kottukal & 36,527 & $3280(8.9 \%)$ & $479(14.6 \%)$ \\
\hline Kuttichal & 20,012 & $946(4.7 \%)$ & $92(9.7 \%)$ \\
\hline Pallichal & 47,118 & $2841(6 \%)$ & $453(15.9 \%)$ \\
\hline Paraniyam & 19,046 & $492(2.5 \%)$ & $199(40.4 \%)$ \\
\hline Poozhanad & 19,253 & $2645(13.7 \%)$ & $299(11.3 \%)$ \\
\hline Thonnakkal & 33,423 & $3468(10.3 \%)$ & $353(10.1 \%)$ \\
\hline Vamanapuram & 28,865 & $1262(4.3 \%)$ & $310(24.5 \%)$ \\
\hline Vattiyoorkavu & 56,830 & $3944(6.9 \%)$ & $396(10 \%)$ \\
\hline
\end{tabular}

* Data excluded from study due to poor quality.

Table 2 compares the demographic, socio-economic and clinical characteristics of all those screened $(N=5307)$ versus the working sample $(N=4527,85.3 \%)$, which excluded those with ungradable or unreliably graded images. The distributions of the variables in the working sample closely matched those of the whole screened population. Seventy percent of the participants were aged between 51 and 70 years and two-thirds of them were females. Ninety-five percent of the participants had only school-level education, although Kerala had a high proportion of enrolment in higher education. Compared to the general population, the sample in this study reported considerably lower income, which is not surprising considering more than half of the sample represented non-workers or housewives. Nearly $75 \%$ of the sample were overweight or obese and $40 \%$ had a waist circumference indicative of central obesity. The blood pressure was normal in only forty percent and the diabetes was uncontrolled in $60 \%$. Neuropathy was the commonest complication after hypertension, while less than $1 \%$ had been told about DR previously. More than a quarter of the patients had insulin as a part of their treatment. Seventy-five percent of the participants had had diabetes for a duration of five years or more.

Table 2. Sample description.

\begin{tabular}{ccc}
\hline Variables & All Screened (100\%) & Working Sample (85.3\%) \\
\cline { 2 - 3 } & $\boldsymbol{N} \mathbf{( \% )}$ & $\boldsymbol{N}$ (\%) \\
\hline Age categories (years) & $5307(100)$ & $4527(100)$ \\
\hline$<30$ & $25(0.5)$ & Excluded \\
\hline $31-40$ & $177(3.3)$ & $142(3.1)$ \\
\hline $41-50$ & $877(16.5)$ & $763(16.9)$ \\
\hline $51-60$ & $1907(35.9)$ & $1691(37.4)$ \\
\hline $61-70$ & $1808(34.1)$ & $1547(34.2)$ \\
\hline
\end{tabular}


Table 2. Cont.

\begin{tabular}{|c|c|c|}
\hline \multirow{2}{*}{ Variables } & All Screened (100\%) & Working Sample (85.3\%) \\
\hline & $N(\%)$ & $N(\%)$ \\
\hline$>70$ & $513(9.7)$ & $384(8.5)$ \\
\hline Gender & $5307(100)$ & $4527(100)$ \\
\hline Female & $3538(66.7)$ & $3023(66.8)$ \\
\hline Male & $1769(33.3)$ & $1504(33.2)$ \\
\hline Education & $5298(100)$ & $4521(100)$ \\
\hline None & $476(9.0)$ & $401(8.9)$ \\
\hline Primary & $2578(48.7)$ & $2159(47.8)$ \\
\hline Secondary & $1943(36.7)$ & $1733(38.3)$ \\
\hline Graduate or higher & $301(5.7)$ & $228(5.0)$ \\
\hline Income & $5016(100)$ & 4275 (100) \\
\hline$<$ INR 600 & $2662(53.1)$ & $2250(52.6)$ \\
\hline$>$ INR 600 & $2354(46.9)$ & $2025(47.4)$ \\
\hline Occupation & $5306(100)$ & 4527 (100) \\
\hline Not Working & $772(14.6)$ & $616(13.6)$ \\
\hline Housewife & $2549(48.0)$ & $2184(48.2)$ \\
\hline Retired & $343(6.5)$ & $295(6.5)$ \\
\hline Unskilled worker & $736(13.9)$ & $652(14.4)$ \\
\hline Skilled Worked & $336(6.3)$ & $282(6.2)$ \\
\hline Professional & $155(2.9)$ & $114(2.5)$ \\
\hline Self employed & $415(7.8)$ & $384(8.5)$ \\
\hline Healthy lifestyle score (Median = 1.5) & $5204(100)$ & $4450(100)$ \\
\hline Below median & $2664(51.2)$ & $2333(52.4)$ \\
\hline Above median & $2540(48.8)$ & $2117(47.6)$ \\
\hline BMI & $5082(100)$ & $4337(100)$ \\
\hline Normal & $1212(23.9)$ & $1042(24.0)$ \\
\hline Overweight & $2450(48.2)$ & $2078(47.9)$ \\
\hline Obese & $1420(27.9)$ & $1217(28.1)$ \\
\hline Waist circumference & $5177(100)$ & $4402(100)$ \\
\hline Below WHO cut-off & $3124(60.3)$ & $2565(58.3)$ \\
\hline Above WHO cut-off & $2053(39.7)$ & $1837(41.7)$ \\
\hline Systolic Blood Pressure & $5223(100)$ & $4458(100)$ \\
\hline$\leq 129 \mathrm{mmHg}$ & $1997(38.2)$ & $1764(39.6)$ \\
\hline $130-139 \mathrm{mmHg}$ & $1307(25.0)$ & $1117(25.1)$ \\
\hline$\geq 140 \mathrm{mmHg}$ & 1919 (36.7) & $1577(35.4)$ \\
\hline Known history of Neuropathy & $5307(100)$ & $4527(100)$ \\
\hline No & $3739(70.0)$ & $3245(71.7)$ \\
\hline Yes & $1568(30.0)$ & $1282(28.3)$ \\
\hline Known history of Diabetic kidney disease & $5307(100)$ & $4527(100)$ \\
\hline No & $5182(97.6)$ & 4419 (97.6) \\
\hline
\end{tabular}


Table 2. Cont.

\begin{tabular}{|c|c|c|}
\hline \multirow{2}{*}{ Variables } & All Screened (100\%) & Working Sample (85.3\%) \\
\hline & $N(\%)$ & $N(\%)$ \\
\hline Yes & $125(2.4)$ & $108(2.4)$ \\
\hline Parental history of Diabetes & $4462(100)$ & $3788(100)$ \\
\hline None & $2312(51.8)$ & $1974(52.1)$ \\
\hline Either or both parents are diabetic & $2150(48.2)$ & $1814(47.9)$ \\
\hline Duration of diabetes & $5307(100)$ & $4527(100)$ \\
\hline$<4$ years & $1322(24.9)$ & $1103(24.4)$ \\
\hline 4 to 9 years & $1775(33.5)$ & $1530(33.8)$ \\
\hline$>9$ years & $2210(41.6)$ & $1894(41.8)$ \\
\hline Insulin used in treatment & $5305(100)$ & 4525 (100) \\
\hline No & $3883(73.2)$ & $3303(73.0)$ \\
\hline Yes & $1422(26.8)$ & $1222(27.0)$ \\
\hline $\mathrm{FPG} \geq 7 \mathrm{mmol} / \mathrm{L}$ or $\mathrm{RBG} \geq 11.1 \mathrm{mmol} / \mathrm{L}$ & $4559(100)$ & $3934(100)$ \\
\hline No & $1814(39.8)$ & $1540(39.2)$ \\
\hline Yes & $2745(60.2)$ & $2394(60.9)$ \\
\hline Self-reported previous diagnosis of DR & $5307(100)$ & $4527(100)$ \\
\hline No & $5265(99.2)$ & 4497 (99.3) \\
\hline Yes & $42(0.8)$ & $30(0.7)$ \\
\hline Family Health Centre & $5307(100)$ & $4527(100)$ \\
\hline Amachal & $140(2.6)$ & $130(2.9)$ \\
\hline Aruvikkara & $412(7.8)$ & $401(8.9)$ \\
\hline Balaramapuram & $538(10.1)$ & $487(10.8)$ \\
\hline Chemmaruthi & $323(6.1)$ & $269(5.9)$ \\
\hline Kadakampalli & $306(5.8)$ & Excluded \\
\hline Karakulam & $353(6.7)$ & $318(7.0)$ \\
\hline Keezhattingal & $264(5.0)$ & $255(5.6)$ \\
\hline Kilimanoor & $390(7.4)$ & $331(7.3)$ \\
\hline Kottukal & $479(9.0)$ & $475(10.5)$ \\
\hline Kuttichal & $92(1.7)$ & $73(1.6)$ \\
\hline Pallichal & $453(8.5)$ & $394(8.7)$ \\
\hline Paraniyam & $199(3.8)$ & $182(4.0)$ \\
\hline Poozhanad & $299(5.6)$ & $263(5.8)$ \\
\hline Thonnakkal & $353(6.7)$ & $312(6.9)$ \\
\hline Vamanapuram & $310(5.8)$ & $255(5.6)$ \\
\hline Vattiyoorkavu & $396(7.5)$ & $382(8.4)$ \\
\hline
\end{tabular}

Abbreviations: FBG—fasting blood glucose; RBG—random blood glucose; BMI—body mass index; INR—Indian rupees; WHO-World Health Organization.

\subsection{Prevalence of $D R$}

The prevalence of any DR was $17.4 \%$ (95\% CI $15.1 \%, 19.7 \%)$ and STDR had a prevalence of $3.3 \%$ (95\%CI $2.1 \%, 4.5 \%$ ) (Table 3). The prevalence of DMO was $2.3 \%(95 \%$ CI $1.3 \%, 3.3 \%)$ suggesting that nearly two-thirds of STDR was contributed by DMO. Adding severe NPDR 
to STDR, the prevalence of DR referable to secondary or tertiary care was $8.3 \%$ ( $95 \% \mathrm{CI}$ $6.3 \%, 10.1 \%$ ), nearly half the cases with any DR.

The age distribution of any DR was ' $n$ ' shaped with lower prevalence for the extreme age ranges of 31-40 years and 70+ years. (Table 3) The lowest prevalence was seen in the category aged 70 years or above. Both STDR and referable DR followed the same distribution. In contrast, DMO showed a declining trend with age (Chi-square for trend $\mathrm{df}=1,11.245, p<0.001)$. The prevalence of any DR was higher in males $(\mathrm{t}=3.87, p=0.002)$. Other outcomes did not show this distinction. Age- and gender-standardised prevalence of DR in each FHC is shown in Table A1.

Table 3. Age- and gender-standardised prevalence of DR $(N=4527)$.

\begin{tabular}{|c|c|c|c|c|}
\hline & Any DR & STDR & DMO & Referable DR \\
\hline & $(\%(95 \% C I))$ & $(\%(95 \% C I))$ & $(\%(95 \% \mathrm{CI}))$ & $(\%(95 \% C I))$ \\
\hline Overall & $17.4(15.1,19.7)$ & $3.3(2.1,4.5)$ & $2.3(1.3,3.3)$ & $8.3(6.5,10.1)$ \\
\hline \multicolumn{5}{|c|}{ Age group (years) } \\
\hline $31-40$ & $15.6(8.8,22.4)$ & $2.8(0.2,5.4)$ & $2.8(0.2,5.4)$ & $6.4(2.1,10.6)$ \\
\hline $41-50$ & $19.6(15.7,23.4)$ & $3.4(1.7,5.1)$ & $2.6(1.1,4.1)$ & $8.6(5.8,11.4)$ \\
\hline $51-60$ & $18.4(16,20.8)$ & $3.5(2.0,5.0)$ & $2.3(1.2,3.4)$ & $9(7.0,11.0)$ \\
\hline $61-70$ & $17.4(14.7,20)$ & $3.2(2.0,4.3)$ & $2.1(1.1,3.1)$ & $8.5(6.7,10.2)$ \\
\hline$>70$ & $9.9(5.7,14.1)$ & $2.8(0.2,5.4)$ & $2.1(0.0,4.6)$ & $5.1(1.7,8.5)$ \\
\hline \multicolumn{5}{|l|}{ Gender } \\
\hline Female & $16.0(13.4,18.6)$ & $3.2(1.9,4.5)$ & $2.3(1.2,3.4)$ & $7.8(6.0,9.7)$ \\
\hline Male & $20.4(18,22.8)$ & $3.5(2.3,4.7)$ & $2.3(1.3,3.2)$ & $9.3(6.9,11.8)$ \\
\hline
\end{tabular}

Abbreviations: DR—diabetic retinopathy, STDR—sight-threatening retinopathy, DMO—diabetic macular oedema, Referrable DR-referrable diabetic retinopathy.

\subsection{Age- and Gender-Standardised Prevalence of DR in Socio-Demographic Groups}

The prevalence of DR outcome was different according to socio-demographic groups in some instances (Table 4). The prevalence for any DR was $13.6 \%$ (95\%CI 10.0\%, 17.1\%) in the highest education group compared to those who had no education $(18.8 \%, 95 \% \mathrm{CI}$ $11.5 \%, 26.0 \% ; p=0.097)$. When occupation is considered, those in professional group had lower prevalence of DR $(9.8 \%, 95 \%$ CI $2.7 \%, 16.9 \%)$ compared to those not working $(20 \%$, $95 \%$ CI $16.5 \%, 23.4 \% ; p=0.032)$. The prevalence of referable DR was $10.2 \%(95 \%$ CI $8.1 \%$, $12.3 \%)$ in the lower income group compared to the higher income group $(6.6 \%, 95 \% \mathrm{CI}$ $(5.0 \%, 8.2 \% ; p \leq 0.001)$ and also among housewives $(7.7 \%, 95 \% \mathrm{CI} 6.1 \%, 9.4 \%)$ compared to those who were not working $(12 \% 95 \%$ CI $8.1 \%, 15.9 \%, p=0.042)$.

Table 4. Age- and gender-standardised prevalence of DR in socio-demographic groups $(N=4527)$.

\begin{tabular}{|c|c|c|c|c|}
\hline & Any DR & STDR & DMO & Referable DR \\
\hline & $(\%(95 \% C I))$ & $(\%(95 \% C I))$ & $(\%(95 \% C I))$ & $(\%(95 \% C I))$ \\
\hline \multicolumn{5}{|l|}{ Education } \\
\hline None & $18.8(11.5,26.0)$ & $2.8(0.6,5)$ & $2.3(0.3,4.4)$ & $9.6(6.3,13)$ \\
\hline Primary & $17.2(14.4,20.1)$ & $3.4(1.5,5.3)$ & $2.5(0.8,4.1)$ & $8.8(6.3,11.2)$ \\
\hline Secondary & $17.8(15.8,19.8)$ & $3.1(2.3,4.0)$ & $2.1(1.4,2.8)$ & $7.7(5.8,9.6)$ \\
\hline Graduate or higher & $13.6(10.0,17.1)$ & $3.9(0.8,6.9)$ & $2.1(0.0,4.4)^{\mathrm{ns}}$ & $7.1(3.4,10.8)$ \\
\hline \multicolumn{5}{|c|}{ Income (Median = INR 600) } \\
\hline Below median & $18.3(14.9,21.8)$ & $3.8(1.8,5.8)$ & $2.7(1.1,4.3)$ & $10.2(8.1,12.3)$ \\
\hline Above median & $16.4(14.2,18.7)$ & $2.7(1.9,3.6)$ & $2.0(1.2,2.7)$ & $6.6(5.0,8.2)$ \\
\hline
\end{tabular}


Table 4. Cont.

\begin{tabular}{|c|c|c|c|c|}
\hline & Any DR & STDR & DMO & Referable DR \\
\hline & $(\%(95 \% C I))$ & $(\%(95 \% C I))$ & $(\%(95 \% C I))$ & $(\%(95 \% C I))$ \\
\hline \multicolumn{5}{|l|}{ Occupation } \\
\hline Not working & $20(16.5,23.4)$ & $4.2(1.9,6.6)$ & $2.2(1.2,3.1)$ & $12(8.1,15.9)$ \\
\hline Housewife & $17.3(14.0,20.6)$ & $3.5(2.0,4.9)$ & $2.5(1.2,3.9)$ & $7.7(6.1,9.4)$ \\
\hline Retired & $19.4(13.9,25)$ & $3.7(1.1,6.4)$ & $3.1(0.9,5.3)$ & $9.1(4.8,13.4)$ \\
\hline Unskilled worker & $16.5(12.8,20.1)$ & $3.5(1.3,5.6)$ & $2.6(0.7,4.5)$ & $8(4.8,11.2)$ \\
\hline Skilled Worker & $16.3(11.3,21.4)$ & $2.9(0.0,6.2)^{n s}$ & $1.6(0.0,3.9)^{\mathrm{ns}}$ & $7.3(3.2,11.4)$ \\
\hline Professional & $9.8(2.7,16.9)$ & $1.6(0.0,4.0)^{\mathrm{ns}}$ & $0.8(0.0,2.5)^{\mathrm{ns}}$ & $3.2(0.0,7.5)^{n s}$ \\
\hline Self employed & $17.8(15.3,20.3)$ & $1.2(0.0,2.9)^{\mathrm{ns}}$ & $1.2(0.0,2.9)^{\mathrm{ns}}$ & $8.4(6.1,10.7)$ \\
\hline
\end{tabular}

There were no differences in the prevalence of any of the DR outcomes according to the categories of healthy lifestyle score and waist circumference (Table 5). However, in the case of BMI, compared to those with normal BMI $(22.2 \%, 95 \%$ CI 19.1\%, 25.3\%), both overweight $(17.5 \% 95 \% \mathrm{CI} 14.3 \%, 20.7 \% ; p=0.005)$ and obese $(13.2 \% 95 \% \mathrm{CI} 10.7 \%, 15.7 \%$; $p<0.001$ ) showed a significantly lower prevalence of any DR. A similar pattern was seen also for the prevalence of referable DR (overweight $p=0.003$; obese $p<0.001$ ).

Table 5. Age- and gender-standardised prevalence of DR according to lifestyle factors $(N=4527)$.

\begin{tabular}{|c|c|c|c|c|}
\hline & Any DR & STDR & DMO & Referable DR \\
\hline & $(\%(95 \% C I))$ & $(\%(95 \% C I))$ & $(\%(95 \% C I))$ & $(\%(95 \% C I))$ \\
\hline \multicolumn{5}{|c|}{ Healthy lifestyle score (Median = 1.5) } \\
\hline Below median & $17.3(14.5,20.2)$ & $3.8(1.9,5.6)$ & $2.5(0.8,4.3)$ & $8.8(6.0,11.6)$ \\
\hline Above median & $17.4(14.9,20)$ & $2.8(1.4,4.2)$ & $2.1(0.9,3.2)$ & $8.0(6.5,9.4)$ \\
\hline \multicolumn{5}{|l|}{ BMI } \\
\hline Normal & $22.2(19.1,25.3)$ & $4.0(2.3,5.7)$ & $2.4(0.7,4.1)$ & $11.1(8.4,13.8)$ \\
\hline Overweight & $17.5(14.3,20.7)$ & $3.1(1.7,4.4)$ & $2.1(1.2,3)$ & $8.0(6.0,10.1)$ \\
\hline Obese & $13.2(10.7,15.7)$ & $2.7(1.2,4.3)$ & $2.4(1.1,3.7)$ & $5.7(4.1,7.3)$ \\
\hline \multicolumn{5}{|l|}{ Waist circumference } \\
\hline Below WHO cut-off & $18.7(15.1,22.3)$ & $3.7(1.8,5.6)$ & $2.7(1.1,4.2)$ & $9.4(6.5,12.2)$ \\
\hline Above WHO cut-off & $15.3(13.4,17.2)$ & $2.5(1.6,3.4)$ & $1.7(0.9,2.4)$ & $6.8(5.0,8.6)$ \\
\hline \multicolumn{5}{|l|}{ Systolic Blood Pressure } \\
\hline$\leq 129 \mathrm{mmHg}$ & $15.5(12.9,18.0)$ & $2.6(1.1,4.1)$ & $1.9(0.7,3.1)$ & $7.5(5.8,9.3)$ \\
\hline $130-139 \mathrm{mmHg}$ & $17.4(14.8,20.0)$ & $3.5(2.0,5.0)$ & $2.4(1.1,3.7)$ & $8.2(6.1,10.3)$ \\
\hline$\geq 140 \mathrm{mmHg}$ & $20.0(15.6,24.5)$ & $4.0(2.3,5.6)$ & $2.7(1.3,4.0)$ & $9.4(6.9,12.0)$ \\
\hline \multicolumn{5}{|l|}{ History of Neuropathy } \\
\hline No & $16.4(13.3,19.5)$ & $3.0(1.5,4.4)$ & $2.3(1.0,3.5)$ & $6.9(5.2,8.7)$ \\
\hline Yes & $20.1(16.9,23.4)$ & $4.1(2.5,5.6)$ & $2.4(1.3,3.4)$ & $11.9(9.0,14.9)$ \\
\hline \multicolumn{5}{|c|}{ History of diabetic kidney disease } \\
\hline No & $17.3(15.0,19.7)$ & $3.3(2.1,4.5)$ & $2.3(1.3,3.3)$ & $8.2(6.4,10.1)$ \\
\hline Yes & $21.4(11.8,30.9)$ & $3.7(0,10.0)$ & $2.8(1.4,4.3)$ & $12.1(2.4,21.8)$ \\
\hline
\end{tabular}


When we consider clinical association (Table 5), significant differences were seen in those with stage 2 hypertension (systolic blood pressure $\geq 140 \mathrm{mmHg}$ ) for any DR $(p=0.039)$ and neuropathy for referable DR $(p=0.002)$.

Subpopulations based on diabetic-related factors except parental history showed the greatest prevalence of DR (Table 6). The prevalence of any DR was significantly higher in those with a parental history of diabetes compared to those who did not $(19.3 \%$ vs. $16.4 \%$, $p=0.022$ ).

In case of the duration of diabetes all outcomes showed trends towards an increase in the prevalence of DR with increasing duration of diabetes $(p<0.001$ (visualised in Figure 2). The prevalence of all DR outcomes was significantly higher in those on insulin treatment and those who reported a previous diagnosis of DR. In addition, patients whose diabetes was not controlled had a significantly higher prevalence of DR outcomes in comparison to those whose diabetes was controlled ( $p<0.001$ in all comparisons).

Table 6. Age- and sex-standardised prevalence of DR according to diabetes-related factors $(N=4527)$.

\begin{tabular}{|c|c|c|c|c|}
\hline & Any DR & STDR & DMO & Referable DR \\
\hline & $(\%(95 \% C I))$ & $(\%(95 \% C I))$ & $(\%(95 \% \mathrm{CI}))$ & $(\%(95 \% \mathrm{CI}))$ \\
\hline \multicolumn{5}{|c|}{ Parental history of diabetes } \\
\hline None & $16.4(13.6,19.2)$ & $3.1(1.7,4.4)$ & $2.3(1.2,3.4)$ & $8.1(6.3,10.0)$ \\
\hline Either or both parents diabetic & $19.3(16.8,21.8)$ & $3.7(2.1,5.4)$ & $2.5(1.1,3.9)$ & $9.3(7,11.7)$ \\
\hline \multicolumn{5}{|l|}{ Duration of diabetes } \\
\hline$<4$ years & $5.1(3.3,6.9)$ & $1.2(0.2,2.2)$ & $1.0(0.0,2.1)$ & $2.1(0.9,3.3)$ \\
\hline 4 to 9 years & $14(11.3,16.7)$ & $2.5(1.2,3.7)$ & $1.8(0.7,3)$ & $6.3(4.3,8.3)$ \\
\hline$>9$ years & $27.9(24.9,31)$ & $5.2(3.5,7)$ & $3.5(2.3,4.7)$ & $13.8(10.8,16.9)$ \\
\hline \multicolumn{5}{|c|}{ Insulin used in treatment } \\
\hline No & $12.2(10.4,13.9)$ & $2.5(1.3,3.6)$ & $1.8(0.7,2.9)$ & $5.6(4.2,7.0)$ \\
\hline Yes & $31.4(27.3,35.6)$ & $5.6(3.6,7.5)$ & $3.6(2.2,5.0)$ & $15.5(11.4,19.5)$ \\
\hline \multicolumn{5}{|l|}{ Hyperglycaemia } \\
\hline $\mathrm{FPG}<7 \mathrm{mmol} / \mathrm{L}$ or $\mathrm{RBG}<11.1 \mathrm{mmol} / \mathrm{L}$ & $13.6(11.6,15.7)$ & $2.5(0.9,4.2)$ & $1.9(0.4,3.4)$ & $6.5(4.6,8.3)$ \\
\hline $\mathrm{FPG} \geq 7 \mathrm{mmol} / \mathrm{L}$ or $\mathrm{RBG} \geq 11.1 \mathrm{mmol} / \mathrm{L}$ & $20.1(17.2,22.9)$ & $4.0(2.5,5.4)$ & $2.8(1.8,3.9)$ & $10.1(8.2,12.1)$ \\
\hline \multicolumn{5}{|c|}{ Self-reported previous diagnosis of DR } \\
\hline No & $17.2(14.9,19.5)$ & $3.2(2.0,4.4)$ & $2.3(1.3,3.3)$ & $8.1(6.3,9.9)$ \\
\hline Yes & $52.5(40.8,64.2)$ & $16.7(5.8,27.6)$ & $6.8(1.6,12.0)$ & $36.3(23.9,48.7)$ \\
\hline
\end{tabular}

\subsection{DR Associations}

There were only a few significant associations between DR and DMO with sociodemographic variables (Table 7). Of interest, higher education (OR 0.81, 95\% CI 0.66, 0.996) and income (OR 0.66 95\% CI 0.52, 0.85) have protective effects on STDR.

Being on insulin is an indicator of high risk of DR (mild to moderate DR: OR 2.05 95\%CI 1.67, 2.52, severe DR: OR 2.48 95\% CI 2.12, 2.92, and STDR: OR 2.08 95\% CI 1.64, 2.65). Hyperglycaemia, especially indicated by random blood glucose, was significant. Among the diabetic complications, neuropathy (OR 1.43 95\% CI 1.13, 1.80) and diabetic kidney disease (OR 4.38 95\% CI 2.98, 6.46) increased the risk of STDR. Unlike macrovascular complications, both high BMI and waist circumference appeared to be protective. The duration of diabetes was significantly associated with DR and DMO with risks increasing linearly with duration. High systolic blood pressure was significantly associated DR but not with DMO or STDR. There were fewer significant associations between the risk factors and DMO in comparison with DR. These factors were hyperglycaemia indicated by random blood glucose, diabetic kidney disease and duration of diabetes. 


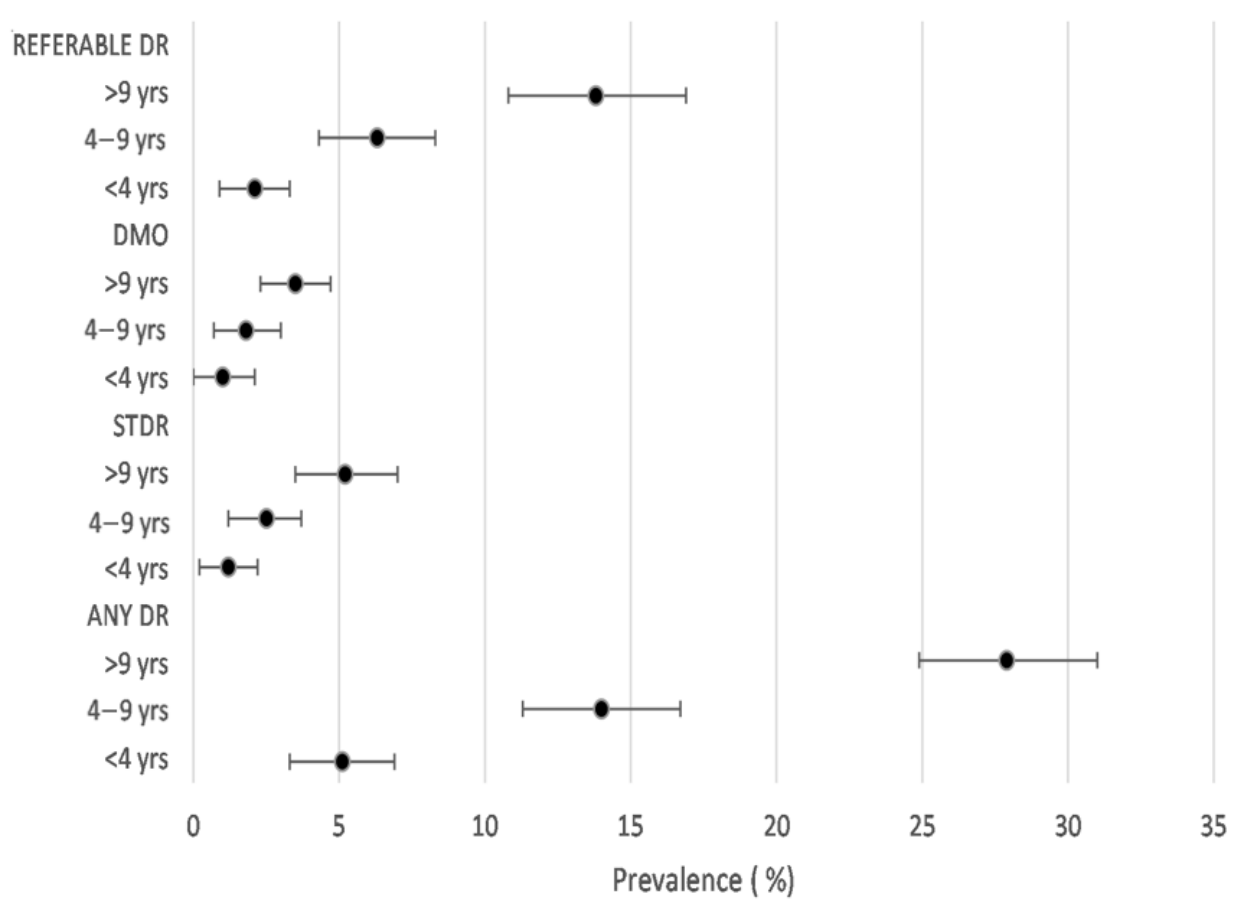

Figure 2. Age- and sex-standardised prevalence of DR outcomes according to duration of diabetes.

Table 7. Associations of DR with mutually adjusted socio-demographic and diabetes-related factors $(N=5307)$.

\begin{tabular}{|c|c|c|c|c|}
\hline & $\begin{array}{c}\text { Any DR } \\
(N=2959)\end{array}$ & $\begin{array}{c}\text { STDR } \\
(N=2959)\end{array}$ & $\begin{array}{c}\text { DMO } \\
(N=2891)\end{array}$ & $\begin{array}{c}\text { Referable DR } \\
(N=2959)\end{array}$ \\
\hline & OR $(95 \% \mathrm{CI})$ & OR $(95 \% \mathrm{CI})$ & OR $(95 \% \mathrm{CI})$ & OR $(95 \% \mathrm{CI})$ \\
\hline \multicolumn{5}{|l|}{ Age group (years) } \\
\hline$<40$ & Reference & Reference & Reference & Reference \\
\hline $41-50$ & $1.25(0.72-2.16)$ & $1.11(0.31-3.9)$ & $0.93(0.29-2.97)$ & $0.91(0.44-1.89)$ \\
\hline $51-60$ & $0.92(0.49-1.74)$ & $0.87(0.21-3.56)$ & $0.65(0.15-2.79)$ & $0.72(0.32-1.62)$ \\
\hline $61-70$ & $0.72(0.39-1.34)$ & $0.57(0.14-2.27)$ & $0.36(0.10-1.23)$ & $0.57(0.26-1.23)$ \\
\hline$>70$ & $0.39(0.17-0.84)$ & $0.91(0.18-4.44)$ & $0.74(0.13-4.26)$ & $0.36(0.15-0.83)$ \\
\hline \multicolumn{5}{|l|}{ Gender } \\
\hline Female & Reference & Reference & Reference & Reference \\
\hline Male & $1.13(0.88-1.46)$ & $1.07(0.53-2.18)$ & $1.07(0.57-2.04)$ & $0.90(0.66-1.27)$ \\
\hline \multicolumn{5}{|l|}{ Education } \\
\hline None & Reference & Reference & Reference & Reference \\
\hline Primary & $1.24(0.76-2.02)$ & $1.03(0.37-2.84)$ & $0.71(0.25-2)$ & $1.03(0.62-1.70)$ \\
\hline Secondary & $1.09(0.65-1.84)$ & $0.82(0.27-2.51)$ & $0.55(0.16-1.85)$ & $0.84(0.46-1.51)$ \\
\hline Graduate or Higher & $1.10(0.64-1.91)$ & $1.55(0.39-6.07)$ & $0.75(0.15-3.72)$ & $1.14(0.55-2.34)$ \\
\hline \multicolumn{5}{|l|}{ Income } \\
\hline Below median & Reference & Reference & Reference & Reference \\
\hline Above median & $0.81(0.63-1.06)$ & $0.61(0.37-0.98)$ & $0.68(0.41-1.14)$ & $0.61(0.46-0.82)$ \\
\hline \multicolumn{5}{|l|}{ Occupation } \\
\hline Not Working & Reference & Reference & Reference & Reference \\
\hline Housewife & $0.95(0.61-1.47)$ & $1.62(0.52-4.98)$ & $2.65(0.75-9.29)$ & $0.75(0.45-1.24)$ \\
\hline Retired & $1.24(0.90-1.72)$ & $1.61(0.58-4.44)$ & $3.51(0.799-15.41)$ & $1.31(0.80-2.13)$ \\
\hline Unskilled Worker & $0.97(0.66-1.41)$ & $1.47(0.51-4.18)$ & $2.30(0.63-8.46)$ & $0.76(0.39-1.48)$ \\
\hline Skilled Worker & $0.88(0.53-1.46)$ & $0.99(0.18-5.39)$ & $1.08(0.12-9.16)$ & $0.73(0.34-1.54)$ \\
\hline
\end{tabular}


Table 7. Cont.

\begin{tabular}{|c|c|c|c|c|}
\hline & $\begin{array}{l}\text { Any DR } \\
(N=2959)\end{array}$ & $\begin{array}{c}\text { STDR } \\
(N=2959)\end{array}$ & $\begin{array}{c}\text { DMO } \\
(N=2891)\end{array}$ & $\begin{array}{l}\text { Referable DR } \\
\quad(N=2959)\end{array}$ \\
\hline & OR $(95 \% \mathrm{CI})$ & OR $(95 \% \mathrm{CI})$ & OR $(95 \% \mathrm{CI})$ & OR $(95 \% \mathrm{CI})$ \\
\hline Professional & $0.50(0.18-1.44)$ & $0.51(0.05-4.93)$ & 1 & $0.43(0.08-2.24)$ \\
\hline Self-Employed & $1.08(0.71-1.62)$ & $0.55(0.09-3.10)$ & $1.11(0.14-8.99)$ & $0.84(0.43-1.65)$ \\
\hline \multicolumn{5}{|l|}{ Lifestyle } \\
\hline \multicolumn{5}{|l|}{ Healthy Lifestyle Score } \\
\hline Below median & Reference & Reference & Reference & Reference \\
\hline Above median & $1.01(0.76-1.34)$ & $0.77(0.32-1.84)$ & $0.90(0.29-2.79)$ & $0.92(0.58-1.46)$ \\
\hline \multicolumn{5}{|l|}{ BMI } \\
\hline Normal & Reference & Reference & Reference & Reference \\
\hline Overweight & $0.82(0.60-1.13)$ & $1.04(0.73-1.49)$ & $1.26(0.82-1.94)$ & $0.70(0.55-0.88)$ \\
\hline Obese & $0.51(0.38-0.69)$ & $0.86(0.46-1.63)$ & $1.18(0.62-2.27)$ & $0.47(0.33-0.67)$ \\
\hline \multicolumn{5}{|l|}{ Waist circumference } \\
\hline Normal & Reference & Reference & Reference & Reference \\
\hline Above WHO cut-off & $0.78(0.62-1)$ & $0.70(0.39-1.24)$ & $0.70(0.35-1.39)$ & $0.83(0.60-1.15)$ \\
\hline \multicolumn{5}{|l|}{ Comorbidities } \\
\hline \multicolumn{5}{|l|}{ Neuropathy } \\
\hline No & Reference & Reference & Reference & Reference \\
\hline Yes & $0.96(0.69-1.32)$ & $1.19(0.67-2.12)$ & $0.84(0.44-1.61)$ & $1.41(1.07-1.85)$ \\
\hline \multicolumn{5}{|l|}{ Chronic Kidney Disease } \\
\hline No & Reference & Reference & Reference & Reference \\
\hline Yes & $0.92(0.54-1.60)$ & $0.53(0.05-5.17)$ & $0.87(0.09-7.90)$ & $0.75(0.28-2.03)$ \\
\hline \multicolumn{5}{|l|}{ Systolic Blood Pressure } \\
\hline$\leq 129 \mathrm{mmHg}$ & Reference & Reference & Reference & Reference \\
\hline $130-139 \mathrm{mmHg}$ & $1.24(0.97-1.59)$ & $1.42(0.71-2.81)$ & $1.28(0.58-3.13)$ & $1.21(0.86-1.71)$ \\
\hline$\geq 140 \mathrm{mmHg}$ & $1.37(1.04-1.80)$ & $1.68(0.87-3.24)$ & $1.50(0.67-3.38)$ & $1.43(1.07-1.91)$ \\
\hline \multicolumn{5}{|l|}{ Diabetes factors } \\
\hline \multicolumn{5}{|l|}{ Positive family history } \\
\hline No & Reference & Reference & Reference & Reference \\
\hline Yes & $1.17(0.91-1.52)$ & $1.27(0.83-1.95)$ & $1.02(0.59-1.75)$ & $1.25(1.01-1.55)$ \\
\hline \multicolumn{5}{|l|}{ Diabetes duration } \\
\hline Less than 4 years & Reference & Reference & Reference & Reference \\
\hline 4 to 9 years & $2.24(1.52-3.29)$ & $1.74(0.86-3.53)$ & $1.87(0.94-3.73)$ & $2.02(1.09-3.74)$ \\
\hline More than 9 years & $4.44(2.64-7.47)$ & $2.58(1.44-4.63)$ & $2.22(1.11-4.44)$ & $3.93(2.04-7.54)$ \\
\hline \multicolumn{5}{|l|}{ Treatment including insulin } \\
\hline No & Reference & Reference & Reference & Reference \\
\hline Yes & $2.54(1.83-3.52)$ & $2.09(1.12-3.92)$ & $1.68(0.64-4.40)$ & $2.71(1.89-3.87)$ \\
\hline \multicolumn{5}{|l|}{ Hyperglycaemia } \\
\hline $\begin{array}{c}\text { FPG }<7 \text { mmol/L) or RBG }<11.1 \\
\mathrm{mmol} / \mathrm{L})\end{array}$ & Reference & Reference & Reference & Reference \\
\hline $\begin{array}{c}\mathrm{FPG} \geq 7 \mathrm{mmol} / \mathrm{L}) \text { or } \mathrm{RBG} \\
\mathrm{mmol} / \mathrm{L})\end{array}$ & $1.38(1.11-1.72)$ & $1.07(0.50-2.28)$ & $1.10(0.47-2.55)$ & $1.26(0.93-1.69)$ \\
\hline
\end{tabular}

Abbreviations: BMI—Body mass Index, DR—diabetic retinopathy, STDR—sight-threatening retinopathy, DMO—diabetic macular oedema,

WHO—World Health Organisation, RBG—random blood glucose, FPG—fasting plasma glucose. 


\section{Discussion}

This study reports the prevalence of DR, STDR, DMO and referable DR in a sample of people with diabetes registered in the NCD register across 15 out of 16 FHCs in the Thiruvananthapuram district in Kerala, where a mydriatic DR teleophthalmology service was set up as a pilot project to evaluate the burden of DR. Primary care in the public health sector is freely accessible but is predominantly accessed by the lower socio-economic strata who cannot afford private healthcare, the major provider of healthcare in India. The study, revealing the prevalence data for the poor, points to the need to ensure access to treatment for persons with DR without catastrophic out-of-pocket payment.

We report that the age- and gender-adjusted prevalence of DR is $17.4 \%$, similar to prevalence data reported from population-based studies in India (range 12-20\%) [21-25]. These figures, although providing less data than those reported in developed countries, are alarming. They show that 3 in 100 people with diabetes who attend the FHCs are at risk of visual impairment due to DR. Furthermore, $8 \%$ required referral to secondary ophthalmic care for further assessment even though only $10 \%$ of the acquired retinal images were ungradable. These results highlight that the burden of eye pathologies other than DR is also high, emphasising the urgent need to establish DR screening and treatment services in the public health system to prevent visual impairment in people with diabetes.

The subpopulations that were more at risk of DR included people aged between 40-70 years, particularly males. This population is the working age group, and visual impairment in this group is likely to have a significant impact on the individual, their family and society. As the public health system is largely accessed by the lower socioeconomic strata and our results show that DR, STDR and DMO are more prevalent in low-income groups, it further underscores the need for systematic screening for DR to be implemented in the public health to prevent health inequity.

There are reports of the urban-rural divide in terms of prevalence of DR in India with rural residents having a lower prevalence of DR [25]. The 16 FHCs covered both urban and rural areas in Thiruvananthapuram district and due to the urbanisation of the whole district, such dissociations are challenging to decipher from this study sample. However, we ensured that the clustering effect of sampling from 16 FHCs were accounted for in our analysis.

Although, the national standards on the proportion of ungradable images in mydriatic DR screening in the more established programme in high-income countries are set at less than 5\% [26], and this teleophthalmology service is set up in low- and middle-income countries (LMIC), where most reports using non-mydriatic retinal imaging show an ungradable rate of less than $30 \%[27,28]$. Cataracts remain a major challenge, and LMIC and DR screening programmes should be used as an opportunity to also identify cataract and other non-DR causes as these conditions are more prevalent than DR [29].

In our study, $8 \%$ of those screened required referral for DR, the greater proportion of which were referred due to severe NPDR rather than STDR, which only accounted for $3.5 \%$ of referrals. However, when we consider the total number of participants that required referral, about $20 \%$ required referral with $10 \%$ being referred due to ungradable images, highlighting that DR screening does detect eye conditions other than STDR that require attention and resources for management [30]. We do not expect all $20 \%$ will require treatment, as some of the ungradable images may be due to the technical failure of not obtaining gradable images [30]. Therefore, these figures may be an over-representation of referable DR.

The sample is representative of the diabetes population at risk of DR because about $75 \%$ had lived more than 4 years with known diabetes, $60 \%$ had uncontrolled diabetes and about a third had systolic BP $\geq 140 \mathrm{mmHg}$ and were already on insulin treatment [31]. About half of the sample had a family history of diabetes, and a third was already known to have another diabetes complication. Therefore, the study sample depicts the people in the low-socio-economic strata in Kerala with several high-risk characteristics of DR, STDR and DMO, highlighting the importance of DR screening in the FHCs. Although men are at 
higher risk of STDR, they are underrepresented (33\%) in this sample. This observation may indicate that males utilise the primary care services less than women.

Another point of consideration is that only $42(0.8 \%)$ of the study participants with DR were aware that they had DR before the study, highlighting the importance of improving public awareness of DR-related blindness and the need for publicly funded systematic DR screening.

The strengths of the study are that it is the first study on DR conducted within the public health system in Kerala on a large population sample. Quality assurance was ensured in the study by repeated training, data monitoring and quality checks. Furthermore, the study is representative of the population attending the FHCs, allowing the extrapolation of the requirements for a publicly funded DR screening programme in the FHCs [32].

The limitations of the study are that although consecutive individuals were meant to be invited to participate in the DR screening programme, the workload of the staff at the FHCs often did not permit such robustness, and hence this is best described as a convenience sample within each FHCs. Therefore, selection bias may have been introduced. These limitations also highlight the difficulties faced in implementing a DR screening programme in the context of resource constraints in a LMIC. However, during the 12month study period, we were able to screen approximately $10 \%$ of the individuals with diabetes registered with the FHCs.

\section{Conclusions}

This study highlights the burden of STDR and its risk factors in the public sector that mainly provides care to people in the low-socio-economic strata. Resources should be allocated to scale up and sustain a state-wide diabetic eye disease screening and treatment programme in Kerala.

Author Contributions: S.S., V.I.S., S.G., R.S., B.G., L.P., D.C., J.S., R.R., R.W., G.N.; Conceptualisation, S.S., G.N., R.W., R.S., B.G., S.G., V.I.S.; methodology, S.S., G.N., R.R., R.S., B.G., V.I.S., S.G., D.C., R.R.; acquisition of data, S.S., G.N., R.W., D.C., R.R., J.S., L.P., B.G., S.M.V.; writing-original draft preparation, S.S., D.C., G.N., R.W., L.P., R.R.; writing-review and editing, S.S., G.N., R.R., R.S., B.G., V.I.S., S.G., D.C., R.R., J.S., L.P., S.M.V.; funding acquisition, S.S., G.N., R.W., R.S., on behalf of the collaborators. All authors have read and agreed to the published version of the manuscript.

Funding: This work was supported by the Global Challenges Research Fund and UK Research and Innovation through the Medical Research Council grant number MR/P027881/1.

Institutional Review Board Statement: The study complied with the Declaration of Helsinki and was approved by The Indian Council of Medical Research (ICMR) and Health Ministry Screening Committee (HMSC / (2018-0551) dated 13 March 2019). Written informed consent was obtained from each participant.

Informed Consent Statement: Written informed consent was obtained from each participant.

Data Availability Statement: The technical text statistical code and dataset will be made available on request after obtaining permission from the Government of Kerala.

Acknowledgments: The authors would like to thank all the study collaborators including the field workers, data operators, staff at the Directorate of Health Services; the Director of Medical Education; the doctors and nurses at family health centres, secondary care and tertiary care centres who were Nayanamritham Project Collaborators and study participants. We also thank Remidio Solutions Ltd. for their staff training.

Conflicts of Interest: The authors declare no conflict of interest.

Patient and Public Involvement: Individuals of the public were involved in the conduct of the study, reporting and dissemination plans of our research. 


\section{Appendix A}

Table A1. Age- and gender-standardised prevalence of DR in each FHC.

\begin{tabular}{|c|c|c|c|c|c|}
\hline & Sample & $\begin{array}{c}\text { Any DR } \\
(\%(95 \% C I))\end{array}$ & $\begin{array}{c}\text { STDR } \\
(\%(95 \% \mathrm{CI}))\end{array}$ & $\begin{array}{c}\text { DMO } \\
(\%(95 \% \mathrm{CI}))\end{array}$ & $\begin{array}{c}\text { Referable DR } \\
(\%(95 \% \mathrm{CI}))\end{array}$ \\
\hline Amachal & 130 & $18.7(18.3,19.1)$ & $3.0(2.9,3.2)$ & $1.5(1.4,1.6)$ & $6.8(6.6,7.0)$ \\
\hline Aruvikkara & 401 & $15.2(15,15.5)$ & $4.2(4.2,4.3)$ & $3(2.9,3)$ & $9.8(9.6,9.9)$ \\
\hline Balaramapuram & 487 & $18.9(18.6,19.2)$ & $2.7(2.6,2.8)$ & $1.2(1.2,1.3)$ & $6(5.9,6.2)$ \\
\hline Chemmaruthi & 269 & $18.5(18.3,18.8)$ & $2.6(2.6,2.6)$ & $2.6(2.6,2.7)$ & $4.5(4.4,4.5)$ \\
\hline Karakulam & 318 & $19.5(19.2,19.7)$ & $3.2(3.1,3.2)$ & $1.6(1.5,1.6)$ & $14.7(14.4,15)$ \\
\hline Keezhattingal & 255 & $19.5(19.2,19.9)$ & $2.3(2.2,2.4)$ & $1.6(1.5,1.7)$ & $6.8(6.6,6.9)$ \\
\hline Kilimanoor & 331 & $13.7(13.5,13.9)$ & $1.8(1.8,1.9)$ & $1.5(1.5,1.6)$ & $3.7(3.6,3.7)$ \\
\hline Kottukal & 475 & $17.2(16.8,17.5)$ & $0.4(0.4,0.4)$ & $0.4(0.4,0.4)$ & $8.3(8.2,8.5)$ \\
\hline Kuttichal & 73 & $25.4(25.1,25.7)$ & $2.7(2.6,2.8)$ & $2.7(2.6,2.8)$ & $12.1(11.8,12.3)$ \\
\hline Pallichal & 394 & $14.8(14.5,15.2)$ & $4.1(4.0,4.2)$ & $1.7(1.7,1.8)$ & $8.5(8.2,8.7)$ \\
\hline Paraniyam & 182 & $17.5(17.2,17.7)$ & $1.6(1.6,1.7)$ & $1.1(1.1,1.1)$ & $9.3(9.2,9.4)$ \\
\hline Poozhanad & 263 & $30.2(29.7,30.7)$ & $9.7(9.3,10)$ & $8.1(7.8,8.4)$ & $15.2(14.8,15.6)$ \\
\hline Thonnakkal & 312 & $19.5(19.2,19.8)$ & $5.4(5.2,5.6)$ & $4.2(4.0,4.4)$ & $10.4(10.1,10.6)$ \\
\hline Vamanapuram & 255 & $10.4(10.2,10.7)$ & $1.6(1.5,1.6)$ & $1.5(1.4,1.6)$ & $6.2(6.0,6.4)$ \\
\hline Vattiyoorkavu & 382 & $12.6(12.4,12.7)$ & $4.4(4.3,4.5)$ & $3.1(3.1,3.2)$ & $6.5(6.4,6.6)$ \\
\hline
\end{tabular}

Abbreviations: DR-Any diabetic retinopathy, STDR-sight-threatening diabetic retinopathy, DMO-diabetic macular oedema, Referable DR-referable diabetic retinopathy.

\section{References}

1. Geldsetzer, P.; Manne-Goehler, J.; Theilmann, M.; Davies, J.I.; Awasthi, A.; Vollmer, S.; Jaacks, L.M.; Bärnighausen, T.; Atun, R. Dia-betes and Hypertension in India: A Nationally Representative Study of 1.3 Million Adults. JAMA Intern. Med. 2018, 178, 363-372. [CrossRef]

2. India State-Level Disease Burden Initiative Diabetes Collaborators. The increasing burden of diabetes and variations among the states of India: The Global Burden of Disease Study 1990-2016. Lancet Glob. Health 2018, 6, e1352-e1362. [CrossRef]

3. Dandona, L.; Dandona, R.; Kumar, G.A.; Shukla, D.K.; Paul, V.K.; Balakrishnan, K.; Prabhakaran, D.; Tandon, N.; Salvi, S.; Dash, A.P.; et al. Nations within a nation: Variations in epidemiological transition across the states of India, 1990-2016 in the Global Burden of Disease Study. Lancet 2017, 390, 2437-2460. [CrossRef]

4. $\quad$ Bourne, R.R.A.; Flaxman, S.R.; Braithwaite, T.; Cicinelli, M.V.; Das, A.; Jonas, J.B.; Keeffe, J.; Kempen, J.H.; Leasher, J.; Limburg, H.; et al. Magnitude, temporal trends, and projections of the global prevalence of blindness and distance and near vision impairment: A system-atic review and meta-analysis. Lancet Glob. Health 2017, 5, e888-e897. [CrossRef]

5. Marmamula, S.; Khanna, R.C.; Kunkunu, E.; Rao, G.N. Population-based assessment of prevalence and causes of visual impair-ment in the state of Telangana, India: A cross-sectional study using the rapid assessment of visual impairment (RAVI) methodology. BMJ Open 2016, 6, e012617.

6. Pearce, E.; Sivaprasad, S. A Review of Advancements and Evidence Gaps in Diabetic Retinopathy Screening Models. Clin. Ophthalmol. 2020, 14, 3285-3296. [CrossRef] [PubMed]

7. Balarajan, Y.; Selvaraj, S.; Subramanian, S. Health care and equity in India. Lancet 2011, 377, 505-515. [CrossRef]

8. Parayil, G. The 'Kerala model' of development: Development and sustainability in the Third World. Third World Q. 1996, 17, 941-958. [CrossRef]

9. Krishnan, G.; Nair, A. Primary health-care innovations with superior allusion to family health centers. Indian J. Community Med. 2021, 46, 149-152. [CrossRef]

10. Nambiar, D.; Sankar, H.; Negi, J.; Nair, A.; Sadanandan, R. Field-testing of primary health-care indicators, India. Bull. World Health Organ. 2020, 98, 747-753. [CrossRef]

11. Mondal, S.; Van Belle, S. India's NCD strategy in the SDG era: Are there early signs of a paradigm shift? Glob. Health 2018, 14, 39. [CrossRef]

12. Sivaprasad, S.; Raman, R.; Conroy, D.; Mohan, V.; Wittenberg, R.; Rajalakshmi, R.; Majeed, A.; Krishnakumar, S.; Prevost, T.; Parameswaran, S.; et al. The ORNATE India Project: United Kingdom- India Research Collabora-tion to tackle visual impairment due to diabetic retinopathy. Eye 2020, 34, 1279-1286. [CrossRef] 
13. Sivaprasad, S.; Netuveli, G.; Wittenberg, R.; Khobragade, R.; Sadanandan, R.; Gopal, B.; Premnazir, L.; Conroy, D.; Srinath, J.; Rama-krishnan, R.; et al. Complex interventions to implement a diabetic retinopathy care pathway in the public health system in Kerala: The Nayanamritham study protocol. BMJ Open 2021, 11, e040577.

14. Natarajan, S.; Jain, A.; Krishnan, R.; Rogye, A.; Sivaprasad, S. Diagnostic Accuracy of Community-Based Diabetic Retinopathy Screening with an Offline Artificial Intelligence System on a Smartphone. JAMA Ophthalmol. 2019, 137, 1182-1188. [CrossRef]

15. Prathiba, V.; Rajalakshmi, R.; Arulmalar, S.; Usha, M.; Subhashini, R.; Gilbert, C.; Anjana, R.; Mohan, V. Accuracy of the smartphone-based nonmydriatic retinal camera in the detection of sight-threatening diabetic retinopathy. Indian J. Ophthalmol. 2020, 68 (Suppl. 1), S42-S46. [CrossRef]

16. Gloucestershire Retinal Education Group. Available online: https:/ /www.gregcourses.com/test-and-training (accessed on 20 October 2021).

17. Wilkinson, C.P.; Ferris, F.L., 3rd; Klein, R.E.; Lee, P.P.; Agardh, C.D.; Davis, M.; Dills, D.; Kampik, A.; Pararajasegaram, R.; Verdaguer, J.T.; et al. Proposed international clinical diabetic retinopathy and diabetic macular edema disease severity scales. Ophthalmology 2003, 110, 1677-1682. [CrossRef]

18. Dolnicar, S.; Grün, B.; Leisch, F. Market Segmentation Analysis. In Market Segmentation Analysis. Management for Professionals; Springer: Singapore, 2018; pp. 11-22. [CrossRef]

19. WHO Expert Consultation. Appropriate body-mass index for Asian populations and its implications for policy and inter-vention strategies. Lancet 2004, 363, 157-163. [CrossRef]

20. Whelton, P.K.; Carey, R.M.; Aronow, W.S.; Casey, D.E., Jr.; Collins, K.J.; Himmelfarb, C.D.; Palma, S.M.D.; Gidding, S.; Jamerson, K.A.; Jones, D.W.; et al. 2017 ACC/AHA/AAPA/ABC/ACPM/AGS/APhA/ASH/ASPC/NMA/PCNA guide-line for the prevention, detection, evaluation, and management of high blood pressure in adults: A report of the American College of Cardiology / American Heart Association Task Force on Clinical Practice Guidelines. Hypertension 2018, 71, $1269-1324$.

21. Raman, R.; Gupta, A.; Krishna, S.; Kulothungan, V.; Sharma, T. Prevalence and risk factors for diabetic microvascular complications in newly diagnosed type II diabetes mellitus. Sankara Nethralaya Diabetic Retinopathy Epidemiology and Molecular Genetic Study (SN-DREAMS, report 27). J. Diabetes its Complicat. 2012, 26, 123-128. [CrossRef]

22. Namperumalsamy, P.; Kim, R.; Vignesh, T.P.; Nithya, N.; Royes, J.; Gijo, T.; Thulasiraj, R.D.; Vijayakumar, V. Prevalence and risk factors for diabetic retinopathy: A population-based assessment from Theni District, south India. Postgrad. Med. J. 2009, 85, 643-648. [CrossRef] [PubMed]

23. Krishnaiah, S.; Das, T.; Nirmalan, P.K.; Shamanna, B.R.; Nutheti, R.; Rao, G.N.; Thomas, R. Risk factors for diabetic retinopathy: Findings from The Andhra Pradesh Eye Disease Study. Clin. Ophthalmol. 2007, 1, 475-482.

24. Rema, M.; Premkumar, S.; Anitha, B.; Deepa, R.; Pradeepa, R.; Mohan, V. Prevalence of Diabetic Retinopathy in Urban India: The Chennai Urban Rural Epidemiology Study (CURES) Eye Study, I. Investig. Ophthalmol. Vis. Sci. 2005, 46, 2328-2333. [CrossRef] [PubMed]

25. Jonas, J.B.; Nangia, V.; Khare, A.; Matin, A.; Bhojwani, K.; Kulkarni, M.; Sinha, A.; Lambat, S.; Gupta, R.; Panda-Jonas, S. Prevalence and Associated Factors of Diabetic Retinopathy in Rural Central India. Diabetes Care 2013, 36, e69. [CrossRef]

26. Scanlon, P.H. The English National Screening Programme for diabetic retinopathy 2003-2016. Acta Diabetol. 2017, 54, 515-525. [CrossRef] [PubMed]

27. Fahadullah, M.; Memon, N.A.; Salim, S.; Ahsan, S.; Fahim, M.F.; Mumtaz, S.N.; Shaikh, S.A.; Memon, M.S. Diagnostic accuracy of non-mydriatic fundus camera for screening of diabetic retinopathy: A hospital based observational study in Pakistan. J. Pak. Med. Assoc. 2019, 69, 378-382. [PubMed]

28. Wadhwani, M.; Vashist, P.; Singh, S.S.; Gupta, N.; Malhotra, S.; Gupta, A.; Shukla, P.; Bhardwaj, A.; Gupta, V. Diabetic retinopathy screening programme utilising non-mydriatic fundus imaging in slum populations of New Delhi, India. Trop. Med. Int. Health 2018, 23, 405-414. [CrossRef]

29. Bartnik, S.E.; Copeland, S.P.; Aicken, A.J.; Turner, A.W. Optometry-facilitated teleophthalmology: An audit of the first year in Western Australia. Clin. Exp. Optom. 2018, 101, 700-703. [CrossRef]

30. Wong, R.L.; Tsang, C.; Wong, D.S.; McGhee, S.; Lam, C.; Lian, J.; Lee, J.W.; Lai, J.S.; Chong, V.; Wong, I.Y. Are we making good use of our public resources? The false-positive rate of screening by fundus photography for diabetic macular oedema. Hong Kong Med. J. 2017, 23, 356-364. [CrossRef]

31. Yau, J.W.Y.; Rogers, S.L.; Kawasaki, R.; Lamoureux, E.L.; Kowalski, J.W.; Bek, T.; Chen, S.-J.; Dekker, J.M.; Fletcher, A.; Grauslund, J.; et al. Global Prevalence and Major Risk Factors of Diabetic Retinopathy. Diabetes Care 2012, 35, 556-564. [CrossRef]

32. Murthy, G.S.; Gilbert, C.; Shukla, R.; Bala, V.; Anirudh, G.; Mukpalkar, S.; Yamarthi, P.; Pendyala, S.; Puppala, A.; Supriya, E.; et al. Overview and project highlights of an initiative to integrate diabetic retinopathy screening and management in the public health system in India. Indian J. Ophthalmol. 2020, 68 (Suppl. 1), S12-S15. [CrossRef] [PubMed] 